\section{(1) \\ CrossMark}

\title{
Pathobiology of pulmonary arterial hypertension: understanding the roads less travelled
}

\author{
Anna R. Hemnes ${ }^{1}$ and Marc Humbert ${ }^{2,3}$
}

Affiliations: ${ }^{1}$ Dept of Medicine, Vanderbilt University Medical Center, Nashville, TN, USA. ${ }^{2}$ Faculté de Médecine, Université Paris-Sud, Université Paris-Saclay, Le Kremlin-Bicêtre, France. ${ }^{3}$ AP-HP, Service de Pneumologie, Centre de Référence de l'Hypertension Pulmonaire, Hôpital Bicêtre, Le Kremlin-Bicêtre, France.

Correspondence: Anna Hemnes, Dept of Medicine, Vanderbilt University Medical Center, 116121 st Ave South, Nashville, TN, 37232, USA. E-mail: anna.r.hemnesđvanderbilt.edu

@ERSpublications

Dysfunction in a multitude of cellular processes plays a critical role in driving the development of PAH http://ow.ly/mGsY30haDXB

Cite this article as: Hemnes AR, Humbert M. Pathobiology of pulmonary arterial hypertension: understanding the roads less travelled. Eur Respir Rev 2017; 26: 170093 [https://doi.org/10.1183/ 16000617.0093-2017].

ABSTRACT The pathobiology of pulmonary arterial hypertension (PAH) is complex and incompletely understood. Although three pathogenic pathways have been relatively well characterised, it is widely accepted that dysfunction in a multitude of other cellular processes is likely to play a critical role in driving the development of PAH. Currently available therapies, which all target one of the three well-characterised pathways, provide significant benefits for patients; however, PAH remains a progressive and ultimately fatal disease. The development of drugs to target alternative pathogenic pathways is, therefore, an attractive proposition and one that may complement existing treatment regimens to improve outcomes for patients. Considerable research has been undertaken to identify the role of the less well-understood pathways and in this review we will highlight some of the key discoveries and the potential for utility as therapeutic targets.

\section{Introduction}

Pulmonary arterial hypertension (PAH) is a progressive and ultimately fatal disease of the pulmonary vasculature. Over the past three decades, there have been major advances in our understanding of the pathobiology of PAH, leading to the development of targeted therapies and improved patient outcomes [1].

The clinical manifestations of PAH result from a reduction in the cross-sectional area of the pulmonary arteries [1], driven by a combination of vasoconstriction, thrombosis, inflammation, and proliferative and obstructive remodelling of the pulmonary artery wall [2]. Like all arteries, the pulmonary artery wall is composed of an outer layer of connective tissue, extracellular matrix (ECM), nerve cells and fibroblasts, and a secondary layer consisting of vascular smooth muscle cells (SMCs) or smooth muscle-like pericytes. Finally, there is a single layer of endothelial cells (ECs), which are in contact with circulating blood [3-5]. The SMCs provide structural integrity and play a crucial role in controlling the diameter of the vessel in

Received: Aug 222017 | Accepted after revision: Dec 082017

Support statement: Funding was received from Actelion Pharmaceuticals Ltd, Allschwil, Switzerland. Funding information for this article has been deposited with the Crossref Funder Registry.

Conflict of interest: Disclosures can be found alongside this article at err.ersjournals.com

Provenance: Publication of this peer-reviewed article was sponsored by Actelion Pharmaceuticals Ltd, Allschwil, Switzerland (principal sponsor, European Respiratory Review issue 146).

Copyright OERS 2017. ERR articles are open access and distributed under the terms of the Creative Commons Attribution Non-Commercial Licence 4.0. 


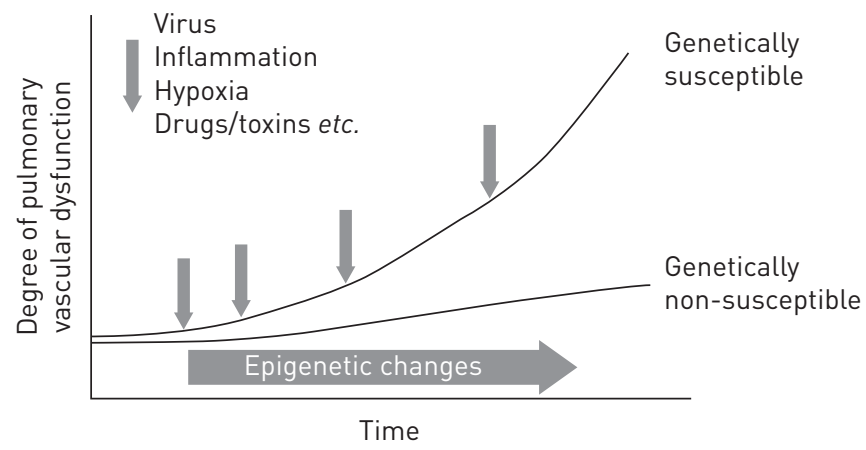

FIGURE 1 A genetic predisposition combined with environmental risk factors may be involved in the development of pulmonary arterial hypertension (PAH). In individuals without mutations in PAH-associated genes (genetically non-susceptible), epigenetic changes and exposure to environmental triggers over time can result in progressive pulmonary vascular dysfunction. This process is accelerated in individuals carrying mutations in PAH-associated genes (genetically susceptible). Reproduced and modified from [8] with permission from the publisher.

response to stimuli [6]. Communication between ECs and SMCs is important for homeostasis within the pulmonary circulation, and can regulate vascular tone and vessel diameter [3-5].

At a molecular level, the pathogenic pathways that drive the development of PAH are still not completely understood. All currently approved PAH therapies target one of three relatively well-characterised pathways (nitric oxide, endothelin and prostacyclin) [1]. However, a multitude of other molecular pathways have been implicated in the pathogenesis of PAH [7], and a genetic predisposition combined with environmental risk factors may be involved in the initial stages of the disease (figure 1) [2]. Intensive research efforts are ongoing to elucidate the complex pathogenic mechanisms that underlie this devastating disease, and this topic will be discussed extensively at the World Symposium on Pulmonary Hypertension in 2018.

The focus of this article is to discuss current research on some of the less well-characterised pathways, from the structural and mechanical changes in the ECM associated with vascular stiffness, to the modification of pulmonary arterial pressure associated with iron deficiency (figure 2, table 1). This article concentrates on pathways of our own particular interest. However, we recognise that there is a wealth of data providing insights into a multitude of other pathogenic pathways in $\mathrm{PAH}$, beyond those covered in this review. Some of these pathways are highlighted in table 2, but are not discussed further in this article. In a timely and relevant review, the metabolic theory of PAH is reviewed comprehensively in the article by ChAn and Rubin [91] in this issue of the European Respiratory Review.

Increasing our understanding of the pathogenic processes underlying the development of PAH provides an opportunity to identify novel therapeutic targets and to expand and improve the treatment options that are available for PAH. Novel therapeutic agents currently in clinical development have recently been reviewed by Simonneau et al. [45] in the European Respiratory Review. In this article, we have highlighted a number of molecular targets that may be of interest for future clinical development (tables 1 and 2).

\section{Vascular stiffness}

Vascular stiffening has been associated with significant morbidity and mortality in a number of vascular diseases [92]. The mechanisms involved include increased wall thickness, SMC proliferation, and changes in the type and quantity of ECM proteins [92]. ECM modifications occur early in the development of $\mathrm{PAH}$ and involve matrix degradation and remodelling, as well as changes in the balance between collagen and elastin deposition [9].

Two recent studies by BERTERO and co-workers $[9,10]$ have provided insights into the molecular pathways involved in vascular stiffening in $\mathrm{PAH}$, and have indicated a role for the co-transcription factors YAP/TAZ (Yes-associated protein/transcriptional coactivator with PDZ-binding motif) and the microRNA-130/301 family (miR-130/301). In 2015, BERTERo et al. [9] demonstrated that ECM remodelling activates YAP/TAZ in pulmonary vascular cells. This induces expression of miR-130/301, which leads to additional ECM remodelling and further up-regulation of YAP/TAZ in a positive feedback loop. A potential pathogenic role for this pathway in pulmonary hypertension $(\mathrm{PH})$ is further supported by observations that inhibition of miR-130/301 is associated with decreased severity of PH in a rat model [9]. In their subsequent study, a relationship between vascular stiffness, YAP/TAZ and metabolic pathways was identified in experimental $\mathrm{PH}$ in rats. Specifically, ECM stiffening, via YAP/TAZ activation, increases the rate of glutaminolysis and production of aspartate, which is required for continued cellular proliferation and migration in stiffened 


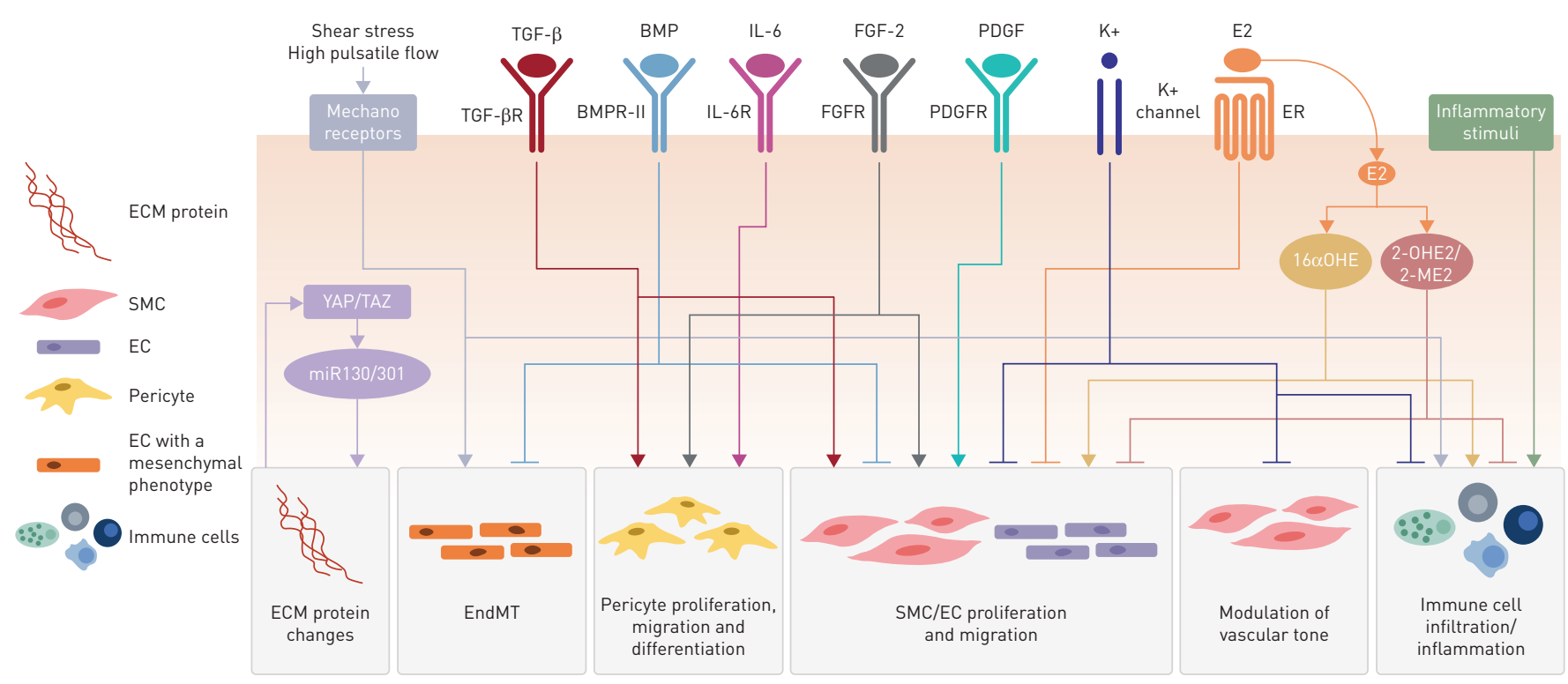

FIGURE 2 Diverse cellular processes and signalling pathways contribute to the pathogenesis of pulmonary arterial hypertension (PAH). Dysfunction in a myriad of overlapping signalling pathways can promote endothelial cell (EC) proliferation and differentiation, smooth muscle cell (SMC) proliferation, migration and vasoconstriction, pericyte proliferation, migration and differentiation, endothelial-to-mesenchymal transition, immune cell infiltration and extracellular matrix (ECM) remodelling in the pulmonary artery. This figure illustrates the pathways and processes discussed in this article and is not an exhaustive illustration of all the pathways currently understood to be involved in the pathogenesis of PAH. EndMT: endothelial-to-mesenchymal transition; TGF- $\beta$ : transforming growth factor- $\beta$; TGF- $\beta$ R: TGF- $\beta$ receptor; BMP: bone morphogenetic protein; BMPR-II: BMP receptor type 2; IL-6: interleukin-6; IL-6R: IL-6 receptor; FGF-2: fibroblast growth factor-2; FGFR: FGF receptor; PDGF: platelet-derived growth factor; PDGFR: PDGF receptor; E2: oestradiol; ER: oestrogen receptor; YAP/TAZ: Yes-associated protein/transcriptional

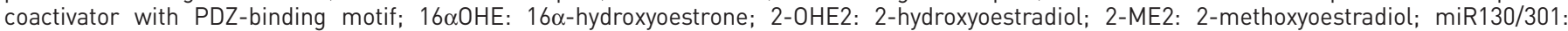
microRNA-130/301 family.

ECM [10]. These studies support the idea that the molecular pathways activated in response to pulmonary vascular stiffening might be a target for novel therapies in $\mathrm{PAH}$.

\section{Endothelial-to-mesenchymal transition}

Severe PAH is associated with the formation of neo-intimal vascular lesions in the pulmonary arteries, caused by increased proliferation of ECs and SMCs. The formation of this neo-intima may involve the process of endothelial-to-mesenchymal transition (EndMT) [11]. In EndMT, ECs acquire a mesenchymal phenotype, a gene expression profile more commonly observed in SMCs, and develop the ability to migrate and remodel the ECM $[11,12]$. A role for EndMT in the development of PAH is supported by the presence of cells with a mixed mesenchymal/endothelial phenotype in intimal lesions from PAH patients, alongside identification of RNA and protein signatures associated with EndMT [13].

Mechanical changes associated with $\mathrm{PAH}$, including high shear stress and high pulsatile flow [12], can be detected by mechanoreceptors on the surface of ECs, leading to expression of proliferative genes $[3,14$, 15] and potentially triggering EndMT [16]. At a molecular level, transforming growth factor (TGF)- $\beta$ has been implicated in the induction of EndMT $[17,18]$, with a reduction in TGF- $\beta$ signalling being associated with reduced neo-intima formation and decreased contribution of endothelial-derived cells to the neo-intima in vivo [17]. Recently, Hopper et al. [11] have provided insights into the mechanism by which TGF- $\beta$ can induce EndMT. They demonstrated, in pulmonary ECs, that dysfunctional signalling through bone morphogenetic protein receptor type 2 (BMPR-II), a member of the TGF- $\beta$ receptor superfamily, leads to increased expression of High Mobility Group AT-Hook 1, which binds to DNA and may promote binding of pro-EndMT transcription factors [11].

There is growing evidence that EndMT occurs in the development of human PAH. Targeting the pathways involved in this process may provide an opportunity to develop anti-remodelling therapies [12].

\section{Pericyte-mediated vascular remodelling}

Pericytes are involved in the stabilisation, maturation and remodelling of vessels, as well as in the regulation of vascular tone and proliferation of ECs $[19,93]$. Abnormal endothelial-pericyte cross-talk can contribute to vascular remodelling [20], and a role for pericytes in the pathogenesis of PAH is supported 
TABLE 1 Pathogenic pathways and potential therapeutic targets in pulmonary arterial hypertension (PAH)

Pathway

Role in PAH

Potential Refs
therapeutic
targets

Vascular stiffness
Endothelial-to-mesenchyma
transition
Pericyte-mediated vascular
remodelling

TGF- $\beta$ signalling

PDGF and FGF signalling

Inflammation and immunity

Resting membrane potential

Oestrogen signalling

Iron homeostasis
Can activate the YAP/TAZ co-transcription factors, leading to further ECM remodelling and modulation of metabolic pathways

Can be induced by haemodynamic changes associated with $\mathrm{PAH}$ TGF- $\beta$ signalling and HMGA1 may play a role in EndMT

EndMT cells can migrate, remodel the ECM and have increased apoptosis resistance

Increased pericyte density in distal pulmonary arteries has been reported in $\mathrm{PAH}$

FGF-2 and IL-6 can stimulate pericyte migration and proliferation

TGF- $\beta$ can promote the differentiation of pericytes into SMCs

Loss of function heterozygous BMPR2 mutations have been reported in $\mathrm{PAH}$

In the absence of a mutation, BMPR-II expression is frequently reduced in $\mathrm{PAH}$

Suppression of BMPR-II signalling leads to increased proliferation and decreased apoptosis in vascular cells

Inflammatory mediators, e.g. TNF- $\alpha$, may play a role in $\mathrm{PAH}$ pathogenesis in the context of BMPR2 mutations

Over-expression of PDGF and FGF has been reported in PAH and may be involved in abnormal proliferation and migration of SMCs, as well as endothelial dysfunction

Inflammatory mediators and cell infiltrates are frequently observed in $\mathrm{PAH}$

Vascular cells can respond to inflammatory stimuli by enhanced proliferation and migration and reduced apoptosis

Loss-of-function KCNK3 mutations have been reported in $\mathrm{PAH}$

May contribute to pulmonary vasoconstriction and pulmonary vascular remodelling

Expression of the Kv1.5 channel is also reduced in human and experimental PAH

E2 metabolites can exert both detrimental and protective effects in $\mathrm{PAH}$

E2 may directly protect against the development of $\mathrm{PH}$ in animal models

Iron deficiency may play a role in pulmonary vascular remodelling

$\begin{array}{ll}\begin{array}{l}\text { Glutaminase } \\ \text { YAP/TAZ }\end{array} & {[9,10]} \\ \text { HMGA1 } & {[11-18]} \\ \text { TGF- } \beta & \\ \text { BMPR-II } & \\ \text { FGF-2 } & {[19,20]} \\ \text { IL-6 } & \\ \text { TGF- } \beta & \\ \text { BMPR-II } & {[21-32]} \\ \text { TNF- } \alpha & \\ \text { SMURF 1 } & \\ \text { miR-140-5p } & \\ & \\ & \\ \text { FGF-2 } & \\ \text { PDGF } & \\ \text { CD20 B } & {[33-44]} \\ \text { IL-1 } \beta \text { and IL-6 } & \\ \text { TNF- } \alpha & \\ \text { KCNK3 } & \\ \text { Kv1.5 } & \end{array}$

miR-29

E2 metabolites

$[68-75]$

Iron replacement

TGF- $\beta$ : transforming growth factor- $\beta$; PDGF: platelet-derived growth factor; FGF: fibroblast growth factor; YAP: Yes-associated protein; TAZ: transcriptional coactivator with PDZ-binding motif; ECM: extracellular matrix; HMGA1: High Mobility Group AT-Hook 1; EndMT: endothelial-to-mesenchymal transition; BMPR-II: bone morphogenic protein receptor type 2; IL: interleukin; SMC: smooth muscle cell; TNF- $\alpha$ : tumour necrosis factor- $\alpha$; SMURF: SMAD-specific E3 ubiquitin protein ligase; miR: microRNA; CD20 B: cluster of differentiation 20 B-lymphocyte antigen; KCNK3: potassium channel subfamily K member 3; Kv1.5: voltage-dependent potassium channel 1.5; E2: oestradiol; $\mathrm{PH}$ : pulmonary hypertension.

by the observation of a two-fold increase in pericyte number in distal pulmonary arteries from the lungs of PAH patients compared with controls [19]. This study also demonstrated that pulmonary EC-derived fibroblast growth factor (FGF)-2 and interleukin-6 can stimulate pericyte migration and proliferation, and that overexpression of TGF- $\beta$ in isolated PAH lung tissue promotes the differentiation of pericytes into SMC-like cells. These responses may potentially contribute to pulmonary vascular remodelling [19]. Therapies targeting the molecular pathways involved in pericyte biology may therefore be potential options for the treatment of PAH.

\section{Growth factor signalling \\ TGF- $\beta$ signalling}

Patients with heritable PAH (HPAH) frequently carry heterozygous mutations in genes encoding receptors of the TGF- $\beta$ superfamily, most commonly BMPR2, which encodes the cell surface receptor BMPR-II [21]. 
TABLE 2 Additional pathogenic pathways and potential therapeutic targets in pulmonary arterial hypertension (PAH)

\begin{tabular}{|c|c|c|c|}
\hline Pathway & Role in PAH & $\begin{array}{c}\text { Potential therapeutic } \\
\text { targets }\end{array}$ & Refs \\
\hline Transcription factors & $\begin{array}{l}\text { FoxO1, a member of the Forkhead box } 0 \text { (FoxO) family of transcription } \\
\text { factors that are key regulators of cellular proliferation, is downregulated } \\
\text { in pulmonary vessels/PASMCs of human/experimental PH lungs } \\
\text { Activation of the prosurvival transcription factors STAT3, NFAT, and } \\
\text { HIF-1 } \alpha \text { has been demonstrated in experimental models of PAH }\end{array}$ & $\begin{array}{l}\text { Fox01 } \\
\text { STAT3/PIM1 } \\
\text { NFAT, HIF-1 } \alpha\end{array}$ & {$[28,82,83]$} \\
\hline $\begin{array}{l}\text { NOTCH3-HES5 } \\
\text { signalling }\end{array}$ & $\begin{array}{l}\text { NOTCH3 is overexpressed in small PASMCs in human PH } \\
\text { There is evidence for a link between NOTCH3 receptor signalling through } \\
\text { HES5 and SMC proliferation in the development of PAH }\end{array}$ & NOTCH3-HES5 pathway & [84] \\
\hline $\begin{array}{l}\text { Epigenetic } \\
\text { mechanisms }\end{array}$ & $\begin{array}{l}\text { Methylation-induced downregulation of SOD2 in PASMCs may create a } \\
\text { metabolic state that favours proliferation and suppresses apoptosis } \\
\text { Aberrant expression of HDACs and BRD4 la transcriptional regulator that } \\
\text { recognises acetylated lysine residues) is consistent with altered } \\
\text { epigenetic mechanisms in PAH } \\
\text { Studies have shown that miRNAs (small RNA molecules that negatively } \\
\text { regulate expression of target genes) are dysregulated in patients with } \\
\text { PAH }\end{array}$ & $\begin{array}{l}\text { SOD2 } \\
\text { HDACs } \\
\text { BRD4 } \\
\text { miRNAs }\end{array}$ & [85-88] \\
\hline VEGFR signalling & $\begin{array}{l}\text { VEGF plasma levels are elevated in patients with severe PAH, and } \\
\text { expression of VEGF and VEGFR2 is robust in complex vascular lesions of } \\
\text { PAH lungs } \\
\text { Role of VEGF in mechanisms of PAH development is not clear }\end{array}$ & VEGFR signalling & [90] \\
\hline \multicolumn{4}{|c|}{$\begin{array}{l}\text { HES5: hairy and enhancer of split 5; PARP-1: Poly [ADP-ribose] polymerase 1; VEGFR: vascular endothelial growth factor receptor; PASMCs: } \\
\text { pulmonary artery smooth muscle cells; PH: pulmonary hypertension; STAT3: Signal transducer and activator of transcription 3; NFAT: nuclear } \\
\text { factor of activated T-cells; HIF-1 } \alpha \text { : hypoxia-inducible factor-1 } \alpha \text {; PIM1: proto-oncogene serine/threonine-protein kinase; SMC: smooth muscle } \\
\text { cell; SOD2: superoxide dismutase 2; HDACs: histone deacetylases; BRD4: bromodomain-containing protein 4; miRNAs: microRNAs; VEGF: } \\
\text { vascular endothelial growth factor. }\end{array}$} \\
\hline
\end{tabular}

Loss-of-function mutations in BMPR2 are present in up to $80 \%$ of HPAH and $11-40 \%$ of idiopathic PAH (IPAH) patients [22]. In the absence of a BMPR2 mutation, reduced pulmonary vascular expression of BMPR-II has also been observed in PAH patients [23]. In a recent study by RoTHMAN et al. [24] the SMAD-specific E3 ubiquitin protein ligase 1 and miR-140-5p were identified as regulators of BMPR-II signalling and may represent a mechanism by which BMPR-II expression is reduced in PAH patients without BMPR2 mutations.

Although the majority of patients with HPAH have heterozygous mutations in the BMPR2 gene, the penetrance of $B M P R 2$ mutations is only $14-42 \%$, indicating that other factors are involved in the pathogenesis of $\mathrm{PAH}$ [25]. Inflammation may be a trigger and this is supported by the observation that the presence of a heterozygous BMPR2 mutation in mice did not lead to $\mathrm{PH}$ in unstressed conditions [26]. However, following introduction of an inflammatory stress, an increase in right ventricular systolic pressure was observed [26]. Another recent study identified a role for tumour necrosis factor (TNF)- $\alpha$ in the development of PH in the context of BMPR-II deficiency [27]. In this study, TNF- $\alpha$-mediated suppression of BMPR-II led to pulmonary artery SMC proliferation. These alterations were confirmed in both an experimental PH animal model and in lung tissue from PAH patients. Furthermore, the introduction of TNF- $\alpha$ to SMCs from isolated HPAH pulmonary artery exacerbates the reduced expression of BMPR-II [27].

As BMPR2 mutations are the most common genetic alteration associated with $\mathrm{PAH}$, it is reasonable to hypothesise that augmenting BMP signalling may provide a therapeutic benefit to patients with PAH. BMPR-II agonists and drugs that non-specifically enhance BMP signalling have shown beneficial effects in experimental PH [24, 28-31]. In cultured ECs, BMP9 was shown to increase BMPR-II signalling, prevent apoptosis, decrease angiogenic behaviour and promote monolayer integrity [31]. Furthermore, administration of etanercept (a TNF- $\alpha$ inhibitor) reversed PH progression in a rat model, restoring BMP signalling and reducing right ventricular systolic pressure, right ventricular hypertrophy and muscularisation of small arterioles [27]. 
Platelet-derived growth factor and FGF signalling

Overexpression of platelet-derived growth factor (PDGF) and FGF has been implicated in the development of PAH [33]. PDGF induces the proliferation and migration of SMCs and fibroblasts [34]. Increased expression of PDGF-A, PDGF-B and their receptors (PDGFR- $\alpha$ and $-\beta$ ) has been reported in the pulmonary arteries of patients with PAH [35]. There are a number of factors that could lead to increased PDGF expression in PAH, including genetic predisposition and increased shear stress $[33,34]$. This may then lead to an undesirable feedback loop, where upregulation of PDGF drives pulmonary vascular remodelling, leading to further shear stress and upregulation of PDGF and PDGFR [33].

Increased FGF-2 levels have been reported in experimental and human PH [36, 37]. FGF-2 is synthesised in a number of cell types, including ECs and fibroblasts, and exerts its influence through FGF receptors, which are expressed on the surface of different cell types, including vascular cells. Overproduction of FGF-2 by pulmonary ECs is thought to be involved in abnormal cross-talk between ECs and SMCs in PAH [36]. Furthermore, EC-derived FGF-2 is overproduced in IPAH and can contribute to the proliferation of cultured SMCs [36]. FGF-2 can also act in an autocrine manner to enhance proliferation and decrease apoptosis of ECs [38].

Given the extensive research on growth factors, there has been much interest in investigating growth factors as therapeutic targets in PAH. A long-term efficacy and safety phase III trial of the PDGFR tyrosine kinase inhibitor imatinib has been performed. However, despite improvements in exercise capacity and haemodynamics observed in patients who tolerated the drug, serious adverse events and premature treatment discontinuations were common [39]. The risks of most tyrosine kinase inhibitors that have been proposed as therapies for PAH (e.g. imatinib, sorafenib) currently outweigh the benefits, although the potential improvement in haemodynamic parameters may warrant further investigation with careful attention to identifying potential markers of a positive clinical response before or early in treatment [40].

\section{Inflammation and immunity}

There is a body of evidence to suggest that altered immune mechanisms play a significant role in the development of PAH via recruitment of inflammatory cells, response of the vascular cells to inflammatory stimuli, and autoimmune responses [46-48]. Inflammatory responses to infectious agents, including viruses and parasites, have also been implicated in the pathogenesis of PAH [49].

Pulmonary vascular lesions identified in patients with PAH, as well as in animal PH models, have been found to contain a number of immune cell types, including $\mathrm{T}$ - and B-lymphocytes, macrophages, dendritic cells and mast cells [50]. Lungs from IPAH patients have also been shown to contain perivascular tertiary lymphoid tissues, consisting of B- and T-cell areas with high endothelial venules and dendritic cells [51]. In IPAH patients, elevated circulating cytokine levels have been reported, some of which are involved in SMC proliferation [52].

Distal ECs respond to high pulsatile flow in arteries with an acute inflammatory response [16] and ECs and SMCs in the pulmonary vasculature can proliferate, migrate or develop an apoptosis-resistant phenotype in response to inflammatory stimuli [46]. Furthermore, pulmonary vascular remodelling can occur in response to inflammatory cytokines [53]. In an experimental model of $\mathrm{PH}$, inflammation associated with CD4 immunodeficiency may be a predisposing factor for $\mathrm{PH}$ development [94]. In support of this, patients with certain PAH-associated conditions, e.g. systemic sclerosis, HIV infection and systemic lupus erythematosus, have previously been shown to exhibit abnormalities in T-regulatory cell number and function [54-56]. This may render these patients more susceptible to pulmonary vascular inflammation and remodelling after initial vascular injury [94]. However, the exact nature of inflammation as a cause or effect is still unknown in IPAH.

Although current PAH treatments possess immunomodulatory properties, there are no approved drugs specifically targeting inflammatory processes. However, a number are being evaluated in clinical trials [45, 57]. Increased inflammatory mediators are frequently observed in the various aetiologies of $\mathrm{PAH}$, but only a small percentage of patients with established PAH (mostly those with active autoimmune diseases) respond to anti-inflammatory drugs [58].

\section{Resting membrane potential}

In 2013, mutations in the potassium channel subfamily $\mathrm{K}$ member 3 (KCNK3 or TASK-1) gene were reported in a family of patients with HPAH [59]. Subsequently, in a case series of 92 patients with HPAH (unrelated to the initial family) and 230 patients with IPAH, loss of function heterozygous mutations in the KCNK3 gene were identified in $3.2 \%$ of the HPAH and $1.3 \%$ of the IPAH patients [59]. A homozygous mutation in the $K C N K 3$ gene has also been reported in a patient with a severe and early form of PAH who did not carry any other mutations known to be associated with PAH [60]. Potassium 
channels can modulate vascular tone through regulation of the resting membrane potential in a number of cell types, including SMCs [61, 62], and may also play a role in the regulation of cell proliferation and migration [63].

The pathophysiological role of KCNK3 in PAH is unclear, but reduced expression and activation is thought to contribute to vasoconstriction and pulmonary artery remodelling via SMC and EC dysfunction [64]. This was suggested in a recent study by ANTIGNy et al. [64] which demonstrated that KCNK3 inhibition in an animal model is associated with increased proliferation of vascular cells, vasoconstriction and inflammation, all of which are known to contribute to PAH progression. Loss of KCNK3 activity is associated with key processes in PAH pathogenesis and it may therefore be a useful therapeutic target. In support of this, ANTIGNY et al. [64] reported that in vivo pharmacological activation of KCNK3 reduced right ventricular systolic pressure, right ventricular hypertrophy and pulmonary vascular wall thickness in a rat model of $\mathrm{PH}$

$\mathrm{PAH}$ is also associated with dysregulation of the potassium channel Kv1.5, which is encoded by the KCNA5 gene. Expression of Kv1.5 is reduced in human and experimental PAH $[65,66]$ and in vivo gene transfer of Kv1.5 was shown to reduce $\mathrm{PH}$ in rats exposed to chronic hypoxia [67]. The mechanisms leading to its reduced expression in $\mathrm{PAH}$ are unclear at the current time.

\section{Oestrogen signalling}

It has long been reported that females are at higher risk of developing PAH compared with males [95]. Indeed, major registry studies have reported marked sex differences, with between 1.8 to 3.9-times as many females included as males [96-102]. For patients with BMPR2 mutations, the risk of developing PAH is strongly influenced by sex: penetrance has been reported to be $14 \%$ for males and $42 \%$ for females in the Vanderbilt Pulmonary Hypertension Registry [25]. The reason for this is unclear, but many studies have implicated sex hormones as mechanistic factors in the development of PAH [95].

Recent interest in the relationship between sex hormones and PAH has been provoked by the observation that oestradiol (E2) metabolites may play a role in PAH pathogenesis. E2 can be converted to oestrone, which is then metabolised to $16 \alpha$-hydroxyoestrone $(16 \alpha \mathrm{OHE})$, a molecule known to have proliferative and inflammatory activities [95]. Alternatively, the CYP1A1 and CYP1B1 enzymes can convert E2 to metabolites such as 2-hydroxyoestradiol (2-OHE2) and 2-methoxyoestradiol (2-ME2), which are known to exert anti-proliferative and anti-inflammatory effects [95]. The differential effects of these metabolites on the development of $\mathrm{PH}$ have been demonstrated in a number of preclinical studies. Chronic exposure of BMPR2 mutant mice to $16 \alpha \mathrm{OHE}$ was associated with increased $\mathrm{PH}$ penetrance and severity compared with mutant mice not exposed to $16 \alpha \mathrm{OHE}$ [68]. Conversely, studies have demonstrated that 2-OHE2 and 2-ME2 may exert protective effects in monocrotaline-induced $\mathrm{PH}$ in rats $[69,70]$. Due to their potentially opposing actions, it has been hypothesised that an imbalance in the production of E2 metabolites may play a role in human PAH pathogenesis. This is supported by Austin et al. [71] who demonstrated that $\mathrm{PAH}$-affected BMPR2 mutation carriers have a lower ratio of urinary 2-OHE2 to $16 \alpha \mathrm{OHE}$ compared with unaffected BMPR2 mutation carriers. This may be due to differences in CYP1B1 activity, as homozygosity for a CYP1B1 genetic polymorphism that has been implicated in several oestrogen-regulated cancers was more common in affected mutation carriers compared with unaffected mutation carriers [71]. These data suggest that if the balance of E2 metabolism shifts towards the production of the pro-proliferative $16 \alpha \mathrm{OHE}$ metabolite, this can increase the risk of PAH in individuals who are already predisposed to the disease.

The exact mechanism by which $16 \alpha \mathrm{OHE}$ can promote the pathogenesis of $\mathrm{PAH}$ remains to be elucidated. However, a recent study by CHEN et al. [72] has indicated a possible role for the miR-29 family. In this study, exposure to $16 \alpha \mathrm{OHE}$ led to increased expression of miR-29 in BMPR2 mutant mice. Furthermore, a beneficial effect of miR-29 antagonism was reported in terms of BMPR-II associated PH penetrance and severity in response to $16 \alpha \mathrm{OHE}$ exposure in these mice. Elevated expression of miR-29 was also observed in lung tissue from HPAH patients, suggesting that this mechanism may also be relevant to the pathogenesis of human PAH. Of note, the miR-29 family has previously been linked to the regulation of glucose and lipid metabolism. This is of particular interest given the known association between PAH and the metabolic syndrome [73].

In addition to the role of its metabolites, E2 itself may directly protect against the development of $\mathrm{PH}$. This hypothesis was explored in a study by LAHM et al. [74] which demonstrated that E2 attenuates the decline in haemodynamic and remodelling parameters observed in hypoxia-induced $\mathrm{PH}$ in rats. In this model, the benefits of E2 are mediated by the oestrogen receptor and conversion to E2 metabolites is not required [74]. However, a protective role for E2 in human PAH has yet to be demonstrated. 
There is now accumulating evidence to support a role for E2 and its metabolites in the pathogenesis of $\mathrm{PH}$. As such, this pathway may be another potential therapeutic target. Despite evidence to support the benefits of $\mathrm{E} 2$ in rat hypoxia-induced $\mathrm{PH}$ [74], the strong female predominance in human PAH makes E2 less appealing as a potential therapy as there are concerns that E2 may promote PAH in humans. Instead, targeting the pathways downstream of E2, e.g. the miR-29 family, may provide a means of treating PAH without the need to modulate sex hormones directly [72].

\section{Iron homeostasis}

The importance of iron status in PAH is illustrated by the fact that iron deficiency is prevalent in patients with IPAH and is associated with disease severity [76-78]. Furthermore, two studies in $\mathrm{PH}$ have demonstrated that the presence of anaemia is indicative of a poor prognosis [103, 104]. In individuals exposed to high altitude, $\mathrm{PH}$ can develop in response to hypoxia [105, 106], and two studies have demonstrated that iron may play a role in this process. In a study by SMITH et al. [79] iron supplementation reduced the increase in pulmonary artery pressure that occurs in response to an acute hypoxic challenge in healthy volunteers. In contrast, iron depletion exacerbated the response of the pulmonary vasculature to hypoxia [79]. A subsequent study by the same authors demonstrated that altitude-induced hypoxic $\mathrm{PH}$ could be attenuated by iron supplementation and exacerbated by iron depletion [80].

Patients can become iron deficient because of reduced intake, impaired uptake or increased loss of iron. In a study by RUITER et al. [77] oral iron therapy was ineffective at restoring normal ferritin levels in eight out of 18 IPAH patients, suggesting that, at least in some patients, a problem with absorption may be responsible for iron deficiency. In support of this, hepcidin, an inhibitor of dietary iron absorption, was shown to be elevated in a population of IPAH patients compared with healthy controls [76]. In the same study, a reduction in BMPR-II expression was associated with an increase in hepcidin expression in vitro. Given that reduced BMPR-II signalling is associated with PAH, this suggests a potential mechanism to explain the hepcidin elevation observed in PAH patients.

Although iron deficiency is prevalent in patients with $\mathrm{PAH}$, the exact relationship between iron and PAH pathogenesis is unknown. A study by Cotroneo et al. [81] has demonstrated for the first time that pulmonary vascular remodelling can occur as a result of chronic iron deficiency in rats. Further experiments indicated that this may result from metabolic changes induced by iron deficiency, including a shift towards aerobic glycolysis [81]. In this animal model, pulmonary vascular remodelling was reversed by iron supplementation, supporting its use as a potential treatment option in PH patients [81].

\section{Conclusions}

In PAH, three well-characterised pathogenic pathways are currently targeted by approved therapies. However, there are many other complex and often interacting pathways involved in the development and progression of the disease. Work is ongoing to elucidate these pathways and increase our understanding of the pathogenesis of PAH. As more data become available on these novel targets, it may become feasible to prioritise which targets should enter clinical development. In recent years, the use of combination therapy to target multiple pathogenic pathways simultaneously has improved outcomes for PAH patients. The development of additional therapies with novel modes of action might further increase our ability to impede pathological changes within the pulmonary vasculature and thus delay the progression of PAH.

\section{Acknowledgements}

The authors would like to thank Claire Lydon (nspm Ltd, Meggen, Switzerland) for medical writing assistance, funded by Actelion Pharmaceuticals Ltd (Allschwil, Switzerland).

\section{References}

1 Humbert M, Lau EM, Montani D, et al. Advances in therapeutic interventions for patients with pulmonary arterial hypertension. Circulation 2014; 130: 2189-2208.

2 Galiè N, Palazzini M, Manes A. Pulmonary arterial hypertension: from the kingdom of the near-dead to multiple clinical trial meta-analyses. Eur Heart J 2010; 31: 2080-2086.

3 Alberts B, Johnson A, Lewis J, et al. Molecular Biology of the Cell. 4th Edn. New York, Garland Science, 2002; p. 1616.

4 Lilly B. We have contact: endothelial cell-smooth muscle cell interactions. Physiology 2014; 29: 234-241.

5 Gao Y, Chen T, Raj JU. Endothelial and smooth muscle cell interactions in the pathobiology of pulmonary hypertension. Am J Respir Cell Mol Biol 2016; 54: 451-460.

6 Metz RP, Patterson JL, Wilson E. Vascular smooth muscle cells: isolation, culture, and characterization. Methods Mol Biol 2012; 843: 169-176.

7 de Jesus Perez VA. Molecular pathogenesis and current pathology of pulmonary hypertension. Heart Fail Rev 2016; 21: 239-257.

8 Tuder R, Archer SL, Dorfmuller P. Relevant issues in the pathology and pathobiology of pulmonary hypertension. J Am Coll Cardiol 2013; 62: Suppl. 25, D4-D12. 
Bertero T, Cottrill KA, Lu Y, et al. Matrix remodeling promotes pulmonary hypertension through feedback mechanoactivation of the YAP/TAZ-miR-130/301 circuit. Cell Rep 2015; 13: 1016-1032.

Bertero T, Oldham WM, Cottrill KA, et al. Vascular stiffness mechanoactivates YAP/TAZ-dependent glutaminolysis to drive pulmonary hypertension. J Clin Invest 2016; 126: 3313-3335.

Hopper RK, Moonen JR, Diebold I, et al. In pulmonary arterial hypertension, reduced BMPR2 promotes endothelial-to-mesenchymal transition via HMGA1 and its target slug. Circulation 2016; 133: 1783-1794.

Stenmark KR, Frid M, Perros F. Endothelial-to-mesenchymal transition: an evolving paradigm and a promising therapeutic target in PAH. Circulation 2016; 133: 1734-1737.

Ranchoux B, Antigny F, Rucker-Martin C, et al. Endothelial-to-mesenchymal transition in pulmonary hypertension. Circulation 2015; 131: 1006-1018.

Chien S. Effects of disturbed flow on endothelial cells. Ann Biomed Eng 2008; 36: 554-562.

Li M, Scott DE, Shandas R, et al. High pulsatility flow induces adhesion molecule and cytokine mRNA expression in distal pulmonary artery endothelial cells. Ann Biomed Eng 2009; 37: 1082-1092.

Elliott WH, Tan Y, Li M, et al. High pulsatility flow promotes vascular fibrosis by triggering endothelial endmt and fibroblast activation. Cell Mol Bioeng 2015; 8: 285-295.

Cooley BC, Nevado J, Mellad J, et al. TGF-beta signaling mediates endothelial-to-mesenchymal transition (EndMT) during vein graft remodeling. Sci Transl Med 2014; 6: 227ra34.

Kumarswamy R, Volkmann I, Jazbutyte V, et al. Transforming growth factor-beta-induced endothelial-tomesenchymal transition is partly mediated by microRNA-21. Arterioscler Thromb Vasc Biol 2012; 32: 361-369.

Ricard N, Tu L, Le Hiress M, et al. Increased pericyte coverage mediated by endothelial-derived fibroblast growth factor-2 and interleukin-6 is a source of smooth muscle-like cells in pulmonary hypertension. Circulation 2014; 129: 1586-1597.

Geevarghese A, Herman IM. Pericyte-endothelial cross-talk: implications and opportunities for advanced cellular therapies. Transl Res 2014; 163: 296-306.

Guignabert C, Bailly S, Humbert M. Restoring BMPRII functions in pulmonary arterial hypertension: opportunities, challenges and limitations. Expert Opin Ther Targets 2017; 21: 181-190.

Fessel JP, Loyd JE, Austin ED. The genetics of pulmonary arterial hypertension in the post-BMPR2 era. Pulm Circ 2011; 1: 305-319.

Atkinson C, Stewart S, Upton PD, et al. Primary pulmonary hypertension is associated with reduced pulmonary vascular expression of type II bone morphogenetic protein receptor. Circulation 2002; 105: 1672-1678.

Rothman AM, Arnold ND, Pickworth JA, et al. MicroRNA-140-5p and SMURF1 regulate pulmonary arterial hypertension. J Clin Invest 2016; 126: 2495-2508.

Larkin EK, Newman JH, Austin ED, et al. Longitudinal analysis casts doubt on the presence of genetic anticipation in heritable pulmonary arterial hypertension. Am J Respir Crit Care Med 2012; 186: 892-896.

Song Y, Jones JE, Beppu $\mathrm{H}$, et al. Increased susceptibility to pulmonary hypertension in heterozygous BMPR2-mutant mice. Circulation 2005; 112: 553-562.

Hurst LA, Dunmore BJ, Long L, et al. TNF $\alpha$ drives pulmonary arterial hypertension by suppressing the BMP type-II receptor and altering NOTCH signalling. Nat Commun 2017; 8: 14079.

Savai R, Al-Tamari HM, Sedding D, et al. Pro-proliferative and inflammatory signaling converge on FoxO1 transcription factor in pulmonary hypertension. Nat Med 2014; 20: 1289-1300.

Spiekerkoetter E, Tian X, Cai J, et al. FK506 activates BMPR2, rescues endothelial dysfunction, and reverses pulmonary hypertension. J Clin Invest 2013; 123: 3600-3613.

Long L, Yang X, Southwood M, et al. Chloroquine prevents progression of experimental pulmonary hypertension via inhibition of autophagy and lysosomal bone morphogenetic protein type II receptor degradation. Circ Res 2013; 112: 1159-1170.

Long L, Ormiston ML, Yang X, et al. Selective enhancement of endothelial BMPR-II with BMP9 reverses pulmonary arterial hypertension. Nat Med 2015; 21: 777-785.

Deng Z, Morse JH, Slager SL, et al. Familial primary pulmonary hypertension (gene PPH1) is caused by mutations in the bone morphogenetic protein receptor-II gene. Am J Hum Genet 2000; 67: 737-744.

Schermuly RT, Dony E, Ghofrani HA, et al. Reversal of experimental pulmonary hypertension by PDGF inhibition. J Clin Invest 2005; 115: 2811-2821.

Humbert M, Monti G, Fartoukh M, et al. Platelet-derived growth factor expression in primary pulmonary hypertension: comparison of HIV seropositive and HIV seronegative patients. Eur Respir J 1998; 11: 554-559.

Perros F, Montani D, Dorfmüller P, et al. Platelet-derived growth factor expression and function in idiopathic pulmonary arterial hypertension. Am J Respir Crit Care Med 2008; 178: 81-88.

Izikki M, Guignabert C, Fadel E, et al. Endothelial-derived FGF2 contributes to the progression of pulmonary hypertension in humans and rodents. J Clin Invest 2009; 119: 512-523.

Benisty JI, McLaughlin VV, Landzberg MJ, et al. Elevated basic fibroblast growth factor levels in patients with pulmonary arterial hypertension. Chest 2004; 126: 1255-1261.

Tu L, Dewachter L, Gore B, et al. Autocrine fibroblast growth factor-2 signaling contributes to altered endothelial phenotype in pulmonary hypertension. Am J Respir Cell Mol Biol 2011; 45: 311-322.

Hoeper MM, Barst RJ, Bourge RC, et al. Imatinib mesylate as add-on therapy for pulmonary arterial hypertension: results of the randomized IMPRES study. Circulation 2013; 127: 1128-1138.

Humbert M. Impression, sunset. Circulation 2013; 127: 1098-1100.

Ghofrani HA, Seeger W, Grimminger F. Imatinib for the treatment of pulmonary arterial hypertension. N Engl $J$ Med 2005; 353: 1412-1413.

Ghofrani HA, Morrell NW, Hoeper MM, et al. Imatinib in pulmonary arterial hypertension patients with inadequate response to established therapy. Am J Respir Crit Care Med 2010; 182: 1171-1177.

Patterson KC, Weissmann A, Ahmadi T, et al. Imatinib mesylate in the treatment of refractory idiopathic pulmonary arterial hypertension. Ann Intern Med 2006; 145: 152-153.

Souza R, Sitbon O, Parent F, et al. Long term imatinib treatment in pulmonary arterial hypertension. Thorax 2006; 61: 736.

Simonneau G, Hoeper MM, McLaughlin V, et al. Future perspectives in pulmonary arterial hypertension. Eur Respir Rev 2016; 25: 381-389. 
Hassoun PM, Mouthon L, Barbera JA, et al. Inflammation, growth factors, and pulmonary vascular remodeling. J Am Coll Cardiol 2009; 54: Suppl. 1, S10-S19.

Voelkel NF, Gomez-Arroyo J, Abbate A, et al. Pathobiology of pulmonary arterial hypertension and right ventricular failure. Eur Respir J 2012; 40: 1555-1565.

Huertas A, Perros F, Tu L, et al. Immune dysregulation and endothelial dysfunction in pulmonary arterial hypertension: a complex interplay. Circulation 2014; 129: 1332-1340.

Kherbeck N, Tamby MC, Bussone G, et al. The role of inflammation and autoimmunity in the pathophysiology of pulmonary arterial hypertension. Clin Rev Allerg Immunol 2013; 44: 31-38.

Rabinovitch M, Guignabert C, Humbert M, et al. Inflammation and immunity in the pathogenesis of pulmonary arterial hypertension. Circ Res 2014; 115: 165-175.

Perros F, Dorfmüller P, Montani D, et al. Pulmonary lymphoid neogenesis in idiopathic pulmonary arteria hypertension. Am J Respir Crit Care Med 2012; 185: 311-321.

Honti G, Brenot F, et al. Increased interleukin-1 and interleukin-6 serum concentrations in severe primary pulmonary hypertension. Am J Respir Crit Care Med 1995; 151: 1628-1631.

Sanchez O, Marcos E, Perros F, et al. Role of endothelium-derived CC chemokine ligand 2 in idiopathic pulmonary arterial hypertension. Am J Respir Crit Care Med 2007; 176: 1041-1047.

Speich R, Jenni R, Opravil M, et al. Primary pulmonary hypertension in HIV infection. Chest 1991; 100: $1268-1271$.

Radstake TR, van Bon L, Broen J, et al. Increased frequency and compromised function of T regulatory cells in systemic sclerosis (SSc) is related to a diminished CD69 and TGF $\beta$ expression. PLoS One 2009; 4: e5981.

Bonelli M, Savitskaya A, Steiner CW, et al. Phenotypic and functional analysis of CD4+ CD25- Foxp3+ T cells in patients with systemic lupus erythematosus. J Immunol 2009; 182: 1689-1695.

Cohen-Kaminsky S, Hautefort A, Price L, et al. Inflammation in pulmonary hypertension: what we know and what we could logically and safely target first. Drug Discov Today 2014; 19: 1251-1256.

Jais X, Launay D, Yaici A, et al. Immunosuppressive therapy in lupus- and mixed connective tissue disease-associated pulmonary arterial hypertension: a retrospective analysis of twenty-three cases. Arthritis Rheum 2008; 58: 521-531.

Ma L, Roman-Campos D, Austin ED, et al. A novel channelopathy in pulmonary arterial hypertension. $N$ Engl J Med 2013; 369: 351-361.

Navas Tejedor P, Tenorio Castano J, Palomino Doza J, et al. An homozygous mutation in KCNK3 is associated with an aggressive form of hereditary pulmonary arterial hypertension. Clin Genet 2017; 91: 453-457.

Brayden JE. Potassium channels in vascular smooth muscle. Clin Exp Pharmacol Physiol 1996; 23: 1069-1076.

Girerd B, Perros F, Antigny F, et al. KCNK3: new gene target for pulmonary hypertension? Expert Rev Respir Med 2014; 8: 385-387.

Huang X, Jan LY. Targeting potassium channels in cancer. J Cell Biol 2014; 206: 151-162.

Antigny F, Hautefort A, Meloche J, et al. Potassium channel subfamily K member 3 (KCNK3) contributes to the development of pulmonary arterial hypertension. Circulation 2016; 133: 1371-1385.

Yuan XJ, Wang J, Juhaszova $\mathrm{M}$, et al. Attenuated $\mathrm{K}+$ channel gene transcription in primary pulmonary hypertension. Lancet 1998; 351: 726-727.

Bonnet S, Michelakis ED, Porter CJ, et al. An abnormal mitochondrial-hypoxia inducible factor-1alpha-Kv channel pathway disrupts oxygen sensing and triggers pulmonary arterial hypertension in fawn hooded rats: similarities to human pulmonary arterial hypertension. Circulation 2006; 113: 2630-2641.

Pozeg ZI, Michelakis ED, McMurtry MS, et al. In vivo gene transfer of the $\mathrm{O}_{2}$-sensitive potassium channel Kv1.5 reduces pulmonary hypertension and restores hypoxic pulmonary vasoconstriction in chronically hypoxic rats. Circulation 2003; 107: 2037-2044.

Fessel JP, Chen X, Frump A, et al. Interaction between bone morphogenetic protein receptor type 2 and estrogenic compounds in pulmonary arterial hypertension. Pulm Circ 2013; 3: 564-577.

Tofovic SP, Salah EM, Mady HH, et al. Estradiol metabolites attenuate monocrotaline-induced pulmonary hypertension in rats. J Cardiovasc Pharmacol 2005; 46: 430-437.

Tofovic SP, Zhang X, Jackson EK, et al. 2-Methoxyestradiol mediates the protective effects of estradiol in monocrotaline-induced pulmonary hypertension. Vascul Pharmacol 2006; 45: 358-367.

Austin ED, Cogan JD, West JD, et al. Alterations in oestrogen metabolism: implications for higher penetrance of familial pulmonary arterial hypertension in females. Eur Respir J 2009; 34: 1093-1099.

Chen X, Talati M, Fessel JP, et al. Estrogen metabolite 16alpha-hydroxyestrone exacerbates bone morphogenetic protein receptor type ii-associated pulmonary arterial hypertension through microrna-29-mediated modulation of cellular metabolism. Circulation 2016; 133: 82-97.

Int J Clin Pract Suppl 2010; 268: 5-13.

Lahm T, Albrecht M, Fisher AJ, et al. 17beta-Estradiol attenuates hypoxic pulmonary hypertension via estrogen receptor-mediated effects. Am J Respir Crit Care Med 2012; 185: 965-980.

Massart J, Sjogren RJO, Lundell LS, et al. Altered miR-29 expression in type 2 diabetes influences glucose and lipid metabolism in skeletal muscle. Diabetes 2017; 66: 1807-1818.

Rhodes CJ, Howard LS, Busbridge M, et al. Iron deficiency and raised hepcidin in idiopathic pulmonary arterial hypertension: clinical prevalence, outcomes, and mechanistic insights. J Am Coll Cardiol 2011; 58: 300-309.

Ruiter G, Lankhorst S, Boonstra A, et al. Iron deficiency is common in idiopathic pulmonary arterial hypertension. Eur Respir J 2011; 37: 1386-1391.

van Empel VP, Lee J, Williams TJ, et al. Iron deficiency in patients with idiopathic pulmonary arterial hypertension. Heart Lung Circ 2014; 23: 287-292.

Smith TG, Balanos GM, Croft QP, et al. The increase in pulmonary arterial pressure caused by hypoxia depends on iron status. J Physiol (Lond) 2008; 586: 5999-6005.

Smith TG, Talbot NP, Privat C, et al. Effects of iron supplementation and depletion on hypoxic pulmonary hypertension: two randomized controlled trials. J Am Med Assoc 2009; 302: 1444-1450.

Cotroneo E, Ashek A, Wang L, et al. Iron homeostasis and pulmonary hypertension: iron deficiency leads to pulmonary vascular remodeling in the rat. Circ Res 2015; 116: 1680-1690. 

Circulation 2014; 129: 786-797.

83 Paulin R, Courboulin A, Meloche J, et al. Signal transducers and activators of transcription-3/pim1 axis plays a critical role in the pathogenesis of human pulmonary arterial hypertension. Circulation 2011; 123: $1205-1215$.

84 Li X, Zhang X, Leathers $\mathrm{R}$, et al. Notch3 signaling promotes the development of pulmonary arterial hypertension. Nat Med 2009; 15: 1289-1297.

85 Archer SL, Marsboom G, Kim GH, et al. Epigenetic attenuation of mitochondrial superoxide dismutase 2 in pulmonary arterial hypertension: a basis for excessive cell proliferation and a new therapeutic target. Circulation 2010; 121: 2661-2671.

Chelladurai P, Seeger W, Pullamsetti SS. Epigenetic mechanisms in pulmonary arterial hypertension: the need for global perspectives. Eur Respir Rev 2016; 25: 135-140.

87 Zhou G, Chen T, Raj JU. MicroRNAs in pulmonary arterial hypertension. Am J Respir Cell Mol Biol 2015; 52: $139-151$.

88 Meloche J, Potus F, Vaillancourt M, et al. Bromodomain-containing protein 4: the epigenetic origin of pulmonary arterial hypertension. Circ Res 2015; 117: 525-535.

89 Federici C, Drake KM, Rigelsky CM, et al. Increased mutagen sensitivity and DNA damage in pulmonary arterial hypertension. Am J Respir Crit Care Med 2015; 192: 219-228.

90 Voelkel NF, Gomez-Arroyo J. The role of vascular endothelial growth factor in pulmonary arterial hypertension. The angiogenesis paradox. Am J Respir Cell Mol Biol 2014; 51: 474-484.

91 Chan SY, Rubin LJ. Metabolic dysfunction in pulmonary hypertension: from basic science to clinical practice. Eur Respir Rev 2017; 26: 170094.

92 Hunter KS, Lammers SR, Shandas R. Pulmonary vascular stiffness: measurement, modeling, and implications in normal and hypertensive pulmonary circulations. Compr Physiol 2011; 1: 1413-1435.

93 Chen Q, Zhang H, Liu Y, et al. Endothelial cells are progenitors of cardiac pericytes and vascular smooth muscle cells. Nat Commun 2016; 7: 12422.

94 Tamosiuniene R, Tian W, Dhillon G, et al. Regulatory $\mathrm{T}$ cells limit vascular endothelial injury and prevent pulmonary hypertension. Circ Res 2011; 109: 867-879.

95 Lahm T, Tuder RM, Petrache I. Progress in solving the sex hormone paradox in pulmonary hypertension. Am $J$ Physiol Lung Cell Mol Physiol 2014; 307: L7-L26.

96 Olsson KM, Delcroix M, Ghofrani HA, et al. Anticoagulation and survival in pulmonary arterial hypertension: results from the COMPERA registry. Circulation 2014; 129: 57-65.

97 Ling Y, Johnson MK, Kiely DG, et al. Changing demographics, epidemiology, and survival of incident pulmonary arterial hypertension: results from the pulmonary hypertension registry of the United Kingdom and Ireland. Am J Respir Crit Care Med 2012; 186: 790-796.

98 Escribano-Subias P, Blanco I, Lopez-Meseguer M, et al. Survival in pulmonary hypertension in Spain: insights from the Spanish registry. Eur Respir J 2012; 40: 596-603.

99 Jing ZC, Xu XQ, Han ZY, et al. Registry and survival study in Chinese patients with idiopathic and familial pulmonary arterial hypertension. Chest 2007; 132: 373-379.

100 Humbert M, Sitbon O, Chaouat A, et al. Pulmonary arterial hypertension in France: results from a national registry. Am J Respir Crit Care Med 2006; 173: 1023-1030.

101 Peacock AJ, Murphy NF, McMurray JJ, et al. An epidemiological study of pulmonary arterial hypertension. Eur Respir J 2007; 30: 104-109.

102 Badesch DB, Raskob GE, Elliott CG, et al. Pulmonary arterial hypertension: baseline characteristics from the REVEAL Registry. Chest 2010; 137: 376-387.

103 Hampole CV, Mehrotra AK, Thenappan T, et al. Usefulness of red cell distribution width as a prognostic marker in pulmonary hypertension. Am J Cardiol 2009; 104: 868-872.

104 Krasuski RA, Hart SA, Smith B, et al. Association of anemia and long-term survival in patients with pulmonary hypertension. Int J Cardiol 2011; 150: 291-295.

105 Ball MK, Waypa GB, Mungai PT, et al. Regulation of hypoxia-induced pulmonary hypertension by vascular smooth muscle hypoxia-inducible factor-1 $\alpha$. Am J Respir Crit Care Med 2014; 189: 314-324.

106 Reeves JT, Grover RF. Insights by Peruvian scientists into the pathogenesis of human chronic hypoxic pulmonary hypertension. J Appl Physiol 2004; 98: 384-389. 